\title{
ATM CONCEPT INTEGRATING TRAJECTORY-ORIENTATION AND AIRBORNE SEPARATION ASSISTANCE IN THE PRESENCE OF TIME- BASED TRAFFIC FLOW MANAGEMENT
}

\author{
Thomas Prevot, Stephen Shelden, Joey Mercer \\ San Jose State University/NASA Ames Research Center, Moffett Field, CA \\ Parimal Kopardekar, \\ Federal Aviation Administration/NASA Ames Research Center, Moffett Field, CA \\ Everett Palmer, Vernol Battiste \\ NASA Ames Research Center, Moffett Field, CA
}

\begin{abstract}
This paper presents a concept for improving National Airspace System (NAS) operations through the adoption of a flexible combination of time-based traffic flow management, trajectoryorientation, and airborne separation assistance elements. Time-based traffic flow management on a NAS-wide and local level assures that local airspace areas are not overloaded at any given time. Trajectory-based operations are used to plan and execute conflict free flight paths for upcoming flight segments. Together, these operations put flight crews in a position to utilize Airborne Separation Assistance Systems (ASAS) to deal with local separation issues, if instructed or permitted by the controller to do so.
\end{abstract}

The concept outlined here is derived from a broad ATM research base, along with first-hand experience and results gathered from within the framework of Distributed Air/Ground - Traffic Management (DAG-TM) research conducted over the last three years. The unique NAS traffic flow management concept proposed here draws on several existing concepts, by combining and modifying them to leverage on their particular strengths. This paper addresses the tools and technologies required and/or desirable for near-term implementation and examines the longer-term implications.

\section{Introduction}

A number of concepts at improving air traffic efficiency and safety have been investigated over past decades. Traditionally, absolute 4D trajectorybased air traffic management and control and relative aircraft-to-aircraft-based spacing concepts have been investigated as alternative pathways. However, research in European programs, such as PHARE [1] and Co-Space [2] and in US programs, such as DAG-TM [3][4] suggest that the combination of these elements is desirable $[5][6][7]$.

Desired capacity, efficiency, and safety gains to cope with future air traffic demand can only be achieved if all layers of the air traffic system including traffic flow management, trajectory planning, and flight execution can accommodate the increased demand. For instance, sophisticated trajectory generation capabilities are of little use if the trajectories can only rarely be flown because of traffic flow management (TFM) constraints or local separation requirements that have not properly been accounted for in the trajectory planning process.

Likewise, a system in which efficient trajectories can only be accommodated by delaying flights on the ground until all elements of the trajectory can be planned conflict-free would not properly account for the highly dynamic nature of the air traffic environment and over-control the problem.

This paper proposes a concept for improving all layers of the air traffic system progressively, thus providing a highly flexible environment that makes effective use of airborne and groundside capabilities.

\section{Layers in the air traffic system}

The air traffic system can be described in detail using layers and feedback loops to describe the hierarchies and interactions between the different components. This method of layers has 
been in use for a long time, but has recently gained new momentum. The reader is referred to [5][8][9] for detailed discussions of the different layers of the air traffic system. For illustration purposes, the following descriptions use simplified views of some of the layers and loops of interest, as they are relevant to show the main differences between the current day situation, a purely trajectory-based approach, and the proposed concept.

\section{Tactical air traffic system}

\section{Description}

Figure 1 shows a simplified view of the interaction between traffic flow management and flight execution. This interaction has been in existence for many years and is still in place for most of today's system. Traffic flow management (TFM) takes NAS host computer data, such as filed flight plans, and some airline inputs to evaluate whether flow constraints need to be applied. Flow constraints are typically relayed to the air traffic controllers as miles-in-trail (MIT) restrictions or meter times. When the flights are executed, air traffic controllers evaluate the local traffic situation within their sector and determine whether the flight is separated sufficiently from other traffic and whether the flow restrictions are met. If an action has to be taken to maintain required separation or to achieve flow conformance, air traffic controllers typically issue tactical heading, altitude, or speed changes to the aircraft, often referred to as radar vectors. If no controller intervention is required, the flights proceed along their filed routings. The flight progress either along the flight plan or along radar vectors is part of the NAS state and is an input parameter to the TFM layer (not explicitly indicated in the Figure 1).

\section{Discussion}

This system relies heavily on the skills of air traffic controllers and traffic flow managers and requires little automation. Most of the long term traffic predictions are made by fairly simple algorithms in the host computer system. Most of the short and medium term predictions are made by controllers and flow managers looking at air traffic displays and mentally extrapolating the situation.

The system is focused on ensuring separation between aircraft within a well structured local traffic problem. With separation management as the primary objective the system has to be considered safe but inefficient.

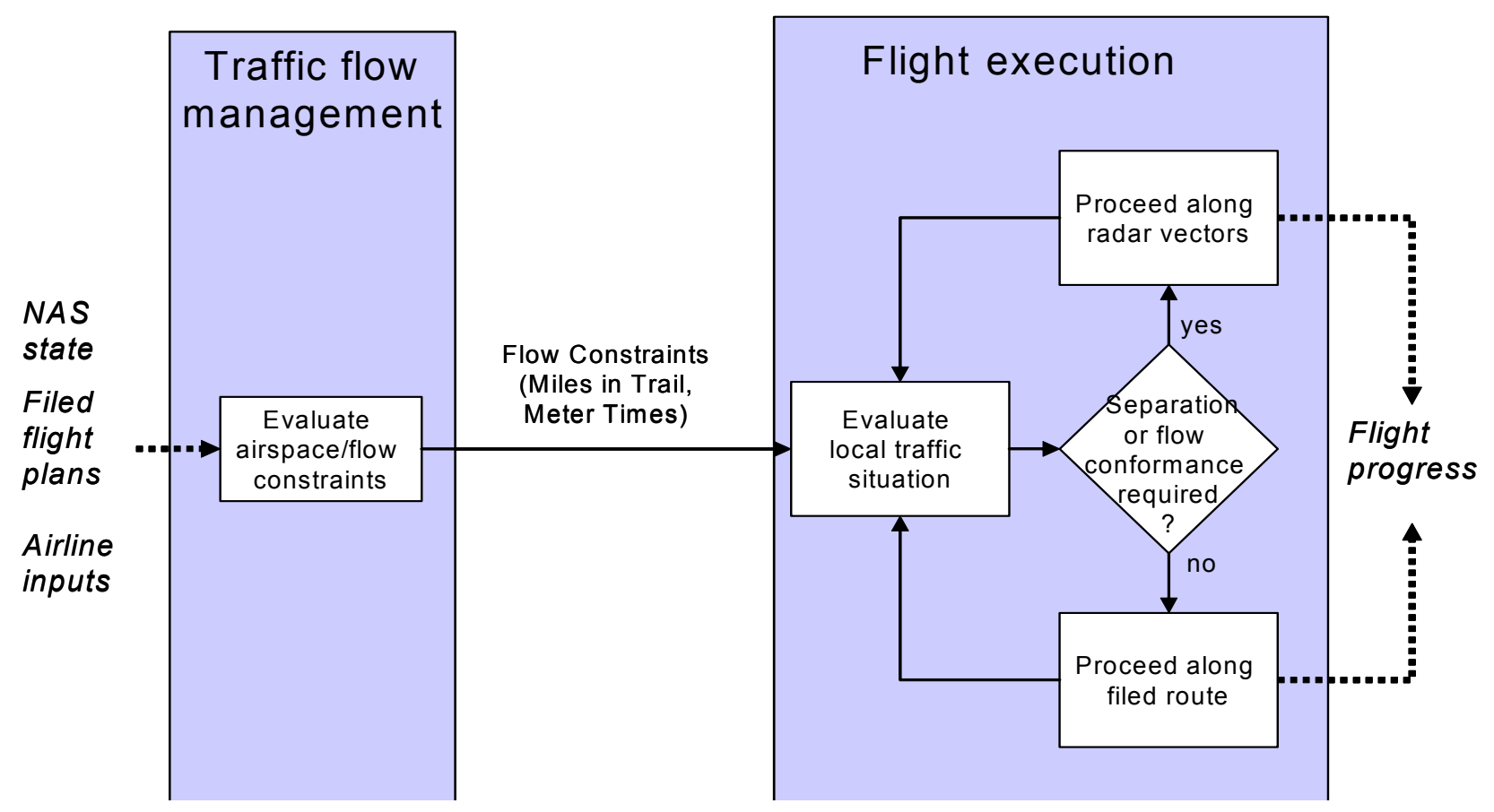

Figure 1: Today's tactical air traffic system is focused on separation management. Traffic flow management provides flow constraints to the flight execution (i. e. air traffic control) layer. 


\section{Strategic trajectory-based system}

\section{Description}

Trajectory-based approaches underlie several research programs like PHARE [1] and Center TRACON Automation System (CTAS) [10] -based concepts such as Active FAST [11] and EDA [12]. The Distributed Air/Ground Traffic Management (DAG-TM) Concept Element (CE) 5 "free maneuvering" and CE 6 "trajectory negotiation" [3] [4] [13] can also be placed into this category. The idea of trajectory-based operations is that each aircraft always proceeds along a conflict free 4D trajectory. In some systems this trajectory is continuously adjusted to the current aircraft state and fed back to the TFM layer. Based on all current trajectories, TFM is now generating time constraints for traffic bottlenecks instead of MIT restrictions. A middle layer for trajectory planning is added between TFM and the flight execution layer that is responsible for generating conflict-free trajectories, which conform to these time constraints.

The trajectory planning process has to consider uncertainties in the environmental conditions caused, for instance, by insufficient weather knowledge and inconsistent trajectory execution. These inconsistencies can be due to different air traffic control and/or piloting techniques as well as imprecise aircraft equipment. The trajectory deconfliction process accounts for prediction uncertainties by adding additional buffers to the required separation between the predicted locations of proximal aircraft. These "bigger than necessary" buffers however reduce the available airspace for planning the trajectories of other flights and can result in inefficient flight paths [7].

\section{Discussion}

The trajectory-based approach has a number of benefits in terms of flight predictability, efficiency, and workload, but it also causes problems, particularly in the areas of trajectory de-confliction and tool capability [3]. The concept poses high requirements on automation capabilities and on communication, navigation, and surveillance (CNS) infrastructure, because conflict detection and resolution need to be highly reliable and trajectories communicated frequently. In [14]Erzberger and Paielli propose an automated airspace concept that heavily relies on automation to generate, communicate, and monitor the trajectories.

It can be expected at least in the short and medium term that automation cannot be used exclusively. Therefore, human automation interaction issues have to be taken into consideration. Frequent changes to the trajectories can cause problems for controllers and pilots who have to evaluate, manipulate and communicate these trajectories. In a purely trajectory-based system, controllers and pilots would have to adjust trajectories for local problems that could otherwise be handled by one or two tactical instructions.

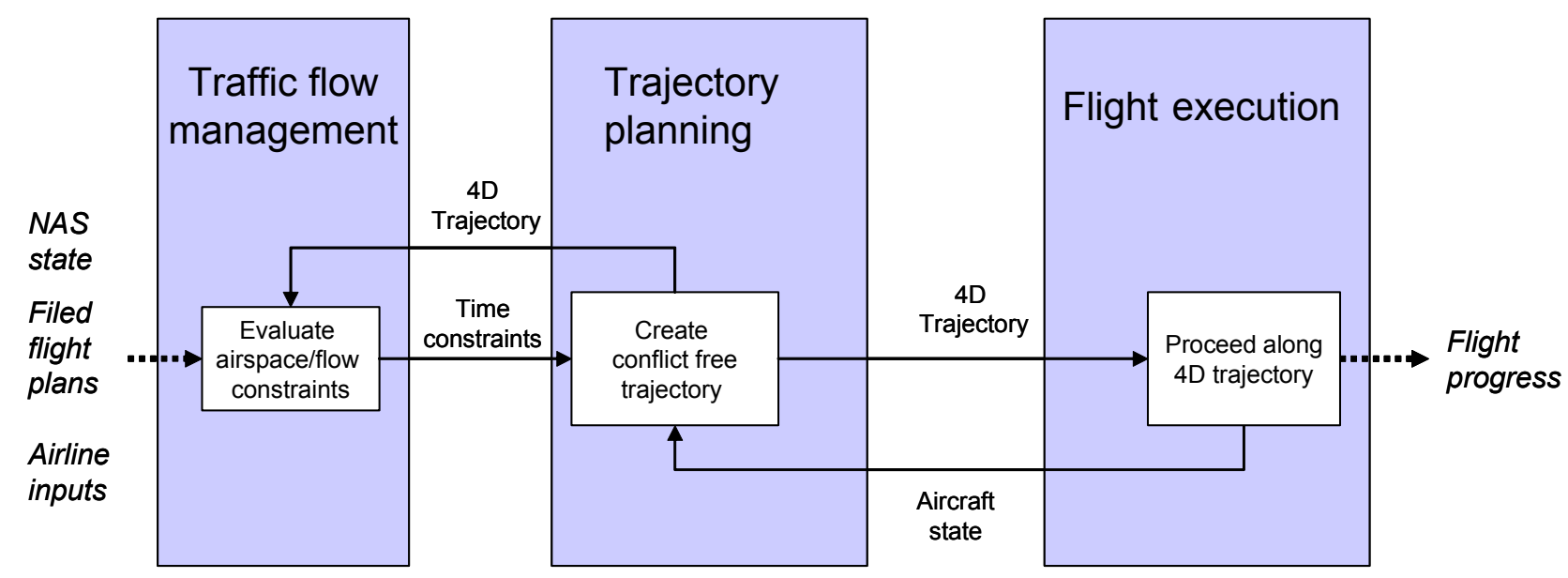

Figure 2 : In a pure trajectory-based system, aircraft should always fly along up to date 4D trajectories that need to be conflict-free and conform to TFM constraints. 


\section{Proposed system}

The above discussion of layers is consistent with the considerations in [5] [6] [7] and other findings (e.g. [15]). It is the common understanding that today's tactical air traffic system is safe, but not as efficient as it could be and will not be able to accommodate the anticipated increase in traffic volume over the next decades unless some fundamental modifications are made. Research on using a trajectory-based system in its pure form has uncovered flight efficiency, predictability, and controller workload benefits at the cost of limited flexibility, potential throughput problems and safety concerns[1][6][7]. In order to realize the "best of both worlds", as discussed in[5] and [6], both approaches can be integrated, as depicted in figure 3.

This paper and others [5] propose a system that is trajectory-based with time-based TFM and a tactical safety layer for achieving local spacing in the flight execution phase. TFM generates a set of time constraints assuring that local airspace areas are not overloaded at any given time, if necessary. Conflict free trajectories are generated that comply with all or at least the upcoming subset of these constraints. If a trajectory that meets the requirements cannot be generated, the preferred trajectory is fed back to TFM to find a new set of time constraints that can be accommodated within the trajectory planning phase. Once a 4D trajectory has been generated, the flight will be executed along the 4D trajectory unless there is a local spacing/separation requirement with another aircraft. In that case, the local situation will be resolved relative to the other aircraft, which may result in a deviation from the 4D trajectory. When the local problem is resolved, the aircraft returns to its trajectory and tries to meet the next time constraint. If the next time constraint cannot be achieved, a new trajectory is created that meets the TFM constraints.

In this system, decision points that trigger trajectory revisions and/or the generation of new time constraints have been introduced. The goal is to minimize the interaction between the layers and create a stable, predictable system.

In the following sections concept details and concrete implementation examples for the different layers will be presented.

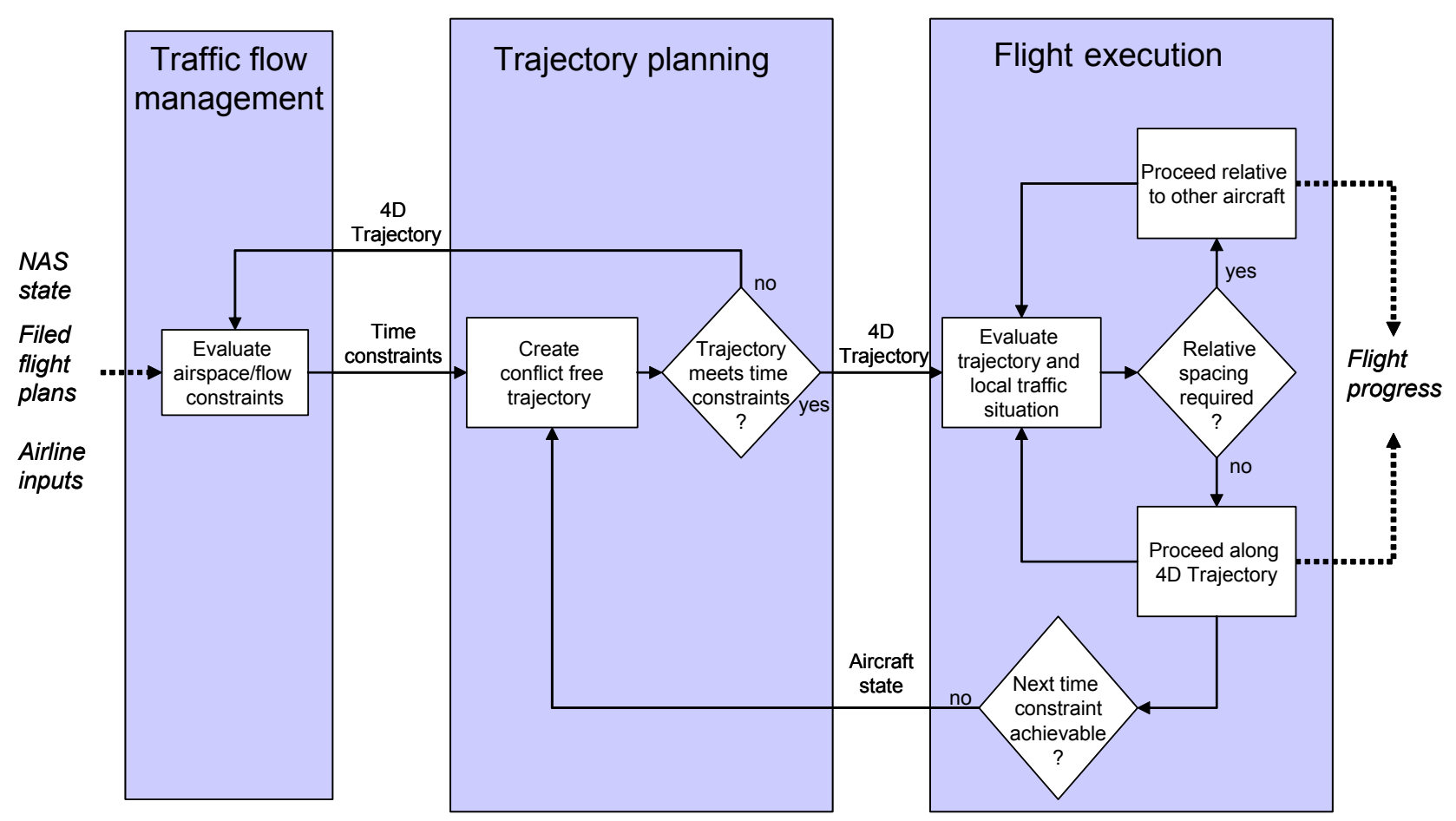

Figure 3: Proposed system: time-based traffic flow management and trajectory-orientation are augmented by a tactical relative spacing loop. Feedback between the layers is event-driven and not continuous 


\section{Concept for time-based traffic flow management}

\section{Miles-in-trail and time-based metering}

The role of TFM in the en route environment is to regulate arrival traffic into terminal areas or any airspace in which traffic demand is expected to exceed capacity. Traffic management coordinators (TMCs) ensure that this does not happen by imposing miles-in-trail (MIT) restrictions that control the flow rate into a constrained area such as an airport. However, MIT restrictions can be inherently inefficient. Thus, time-based metering has been explored. Petre [16] among others described applications of time-based air traffic control in an extended terminal area.

Decision support tools such as the CTAS Traffic Management Advisor (TMA) enable a metering scheme in which aircraft are metered at a fix or arc such that demand will not exceed capacity [17] in the downstream airspace. Time-based metering has significantly improved arrival operations at airports such as Dallas-Ft. Worth that are traditionally capacity-constrained.

\section{Extending time-based TFM to the entire NAS}

By extending the use of time-based metering to the entire NAS, additional efficiency and capacity gains can be expected. Converting TFM operations from distance-based to time-based facilitates trajectory-oriented operations and acknowledges that scheduling is the highest airline priority, which if met would be beneficial for the entire NAS. TMCs could generate schedules based on identified constraints and dynamic airline priorities.

The successful integration of time-based metering into the current environment relies on coordinating operations from multiple facilities. Therefore, Multi-Center TMA (MC-TMA) was developed. Currently, MC-TMA is being tested for Philadelphia arrivals that transition through New York, Boston, Washington, and Cleveland Air Route Traffic Control Centers (ARTCCs) [18]. Based on experiences gathered in Los Angeles ARTCC trials, it appears that this type of timebased metering is acceptable to controllers.
Green [8] described an en route spacing tool to address the current TFM problems into flowrestricted airspace by using conflict probing and trial planning tools to reduce the number of aircraft merge points that are due to the current route structure. One of the major advantages of that spacing tool is the ability to accommodate path independent spacing. Such a tool integrated with time-based metering can support path independent trajectory-based operations with higher efficiency.

\section{Coordination of mixed operations}

The DAG-TM CE 5 "free maneuvering" investigates operations that represent a mixture of ATC-managed and autonomous aircraft. The coordination of these operations in high density airspace imposes additional challenges that can be addressed by time-based TFM. This metering concept can provide scheduled and required times of arrival for managed and autonomous aircraft, thus coordinating their operations. Flight crews of equipped aircraft would meet the required time of arrival using airborne automation. Controllers would use ground-based DSTs to generate required trajectory changes that deliver unequipped aircraft at their STA. Thus, the traffic flow of autonomous and managed aircraft into flow constraint airspace can be coordinated by scheduling alone without imposing additional workload on the air traffic controllers.

In essence, time-based metering would serve as one of the core elements in facilitating operations that use 4-D trajectories and RTAs. Moving forward to advanced concepts such as trajectory orientated free maneuvering or limited delegation, TFM could dynamically create the time-restrictions that regulate the traffic flow according to capacity/demand ratios and airline preferences on an as-needed basis.

The concept for trajectory planning and flight execution to implement these restrictions efficiently is described in the following sections. 


\section{Concept for trajectory planning and flight execution}

\section{Airborne Separation Assistance}

The generic layers described earlier do not make any assumptions about the particular task distribution between the flight crew and the air traffic controllers. However, in order to make the best use of emerging technologies it appears appropriate to utilize Airborne Separation Assistance System (ASAS) concepts that have already shown good potential for relieving the air traffic controller of some of the monitoring tasks related to the tactical safety layer [2].

If ASAS operations are used for localized separation requirements, the set of trajectories need only provide separation buffers that reflect the tolerances, which can be assumed achievable with local ASAS operations. The TFM-conforming trajectory planning can ensure that the airspace is not overloaded at any given time. RTAs can be sent from scheduling tools to meter aircraft into highdensity areas. Crossing, merging, and in-trail following activities would be handled by relative spacing operations. In contrast, passing situations and head on conflicts would be resolved via trajectory changes, since they require route or altitude modifications that are not currently part of ASAS operations.

\section{Concept Definition}

With the ASAS integration the concept for trajectory planning and flight execution that goes along with the system proposed in figure 3 can be defined as follows [5]:

(1) Use trajectory-based operations to create efficient, nominally conflict-free trajectories that conform to traffic management constraints and,

(2) maintain local spacing between aircraft with airborne separation assistance.

It is intended that the concept:

- Take full advantage of the traffic flow management benefits of the trajectoryoriented approach.
- Reduce to a minimum any additional conflict resolution buffers arising out of prediction uncertainty.

- Reduce controller workload.

- Minimally impact flight crew workload.

- Have a positive effect on controller and flight crew traffic awareness.

- Limit the deviations from the 4D path to short-term deviations mostly due to local traffic situations, thereby minimizing the medium to long-term prediction uncertainty.

- Minimize lateral route and/or altitude changes for local separation assurance.

\section{Illustration}

The concept is further illustrated in the context of the hypothetical traffic situation, which is indicated in figure 4. In order to provide for the safe and efficient flow of traffic, the following has to be accomplished [7]:

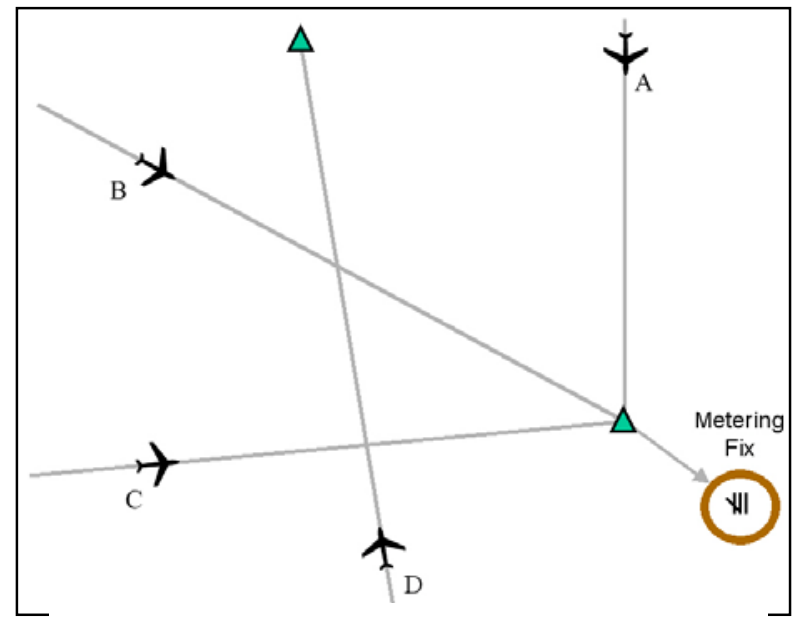

1. Overflight aircraft $\mathrm{D}$ needs to be separated from arrivals $\mathrm{A}$ and $\mathrm{B}$.

2. Arrival aircraft A, B and C need to be delivered to the metering fix, closely spaced, but safely separated. (Assume a target distance of six NM in trail.) 
3. The route changes necessary to accomplish (1) and (2) above need to be minimized, in terms of course deviation and flight crew workload

The traffic problem could be handled as illustrated in Figures 5 and 6.

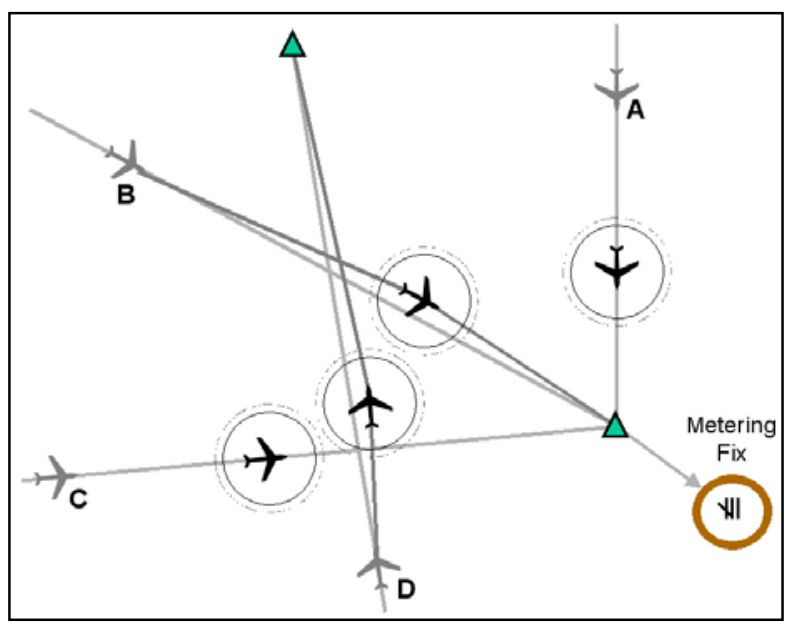

Figure 5: Solution to traffic problem in the crossing phase.

Any excess spacing buffers required for trajectory de-confliction can be eliminated because of the additional safety layer provided by the relative operations with ASAS. The overflight aircraft $\mathrm{D}$ can be planned to pass through the arrivals $\mathrm{B}$ and $\mathrm{C}$ with only a minor route modification for aircraft $B$ and $D$. The schedule at the metering fix can be planned aggressively, because when the aircraft get close at the merge point the relative operations will take over and ensure minimum separation. The schedule can be communicated to the flight deck, which can input the time as an RTA.

Whenever aircraft encounter local spacing problems, relative operations could be used. In figure 5 aircraft D crosses six nautical miles (NM) behind $\mathrm{B}$, and $\mathrm{C}$ crosses six NM behind $\mathrm{D}$. These modifications can be accomplished with minor route changes by the controller and speed changes managed on the flight deck, without overloading the controller. When the separation situation is resolved, aircraft can resume their original trajectories, speeding up or slowing down slightly to make up for the intermediate speed changes or trajectory deviations. Once on time at the next merge point, relative operations can again be used to fine-tune the merge.

In figure $6, \mathrm{~B}$ merges behind $\mathrm{A}$, and $\mathrm{C}$ merges behind $\mathrm{B}$. By delegating the relative spacing task to the flight deck, an efficient and flexible flow will be maintained without increasing the controller's workload.

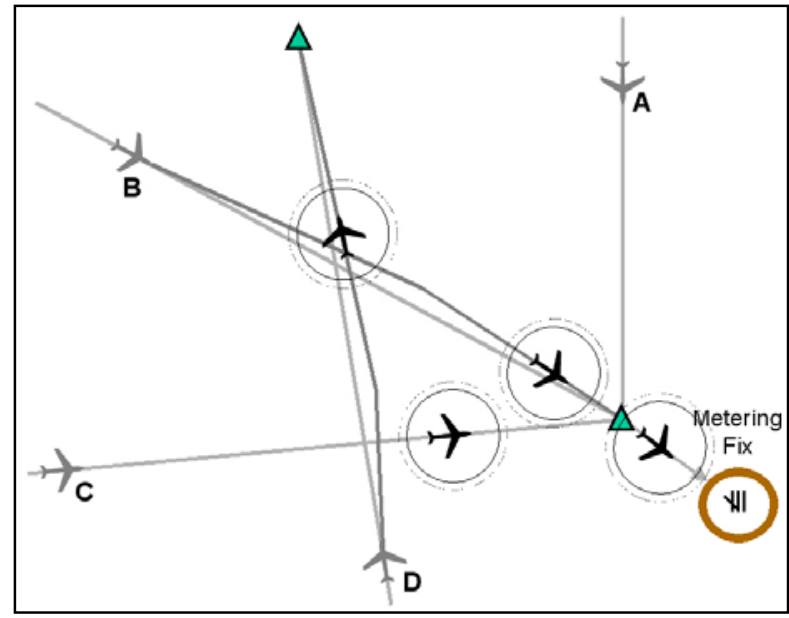

Figure 6: Solution to traffic problem in the merge phase.

In order to apply this concept successfully, the trajectories should be planned with nominal speed profiles that avoid using the edges of the aircraft's operating envelope. This is fuel-efficient and allows room for speed changes for spacing operations.

\section{Implementation}

\section{Near-term: Ground-based trajectory planning and limited delegation of spacing task to properly equipped aircraft}

A near term implementation of the concept can utilize existing ground-based trajectory tools like those provided by CTAS to generate the TFMconforming 4D trajectories. These trajectories can be communicated via Controller Pilot Data Link Communication (CPDLC) to equipped aircraft. These concepts have been investigated and found promising for controllers [6], [20] and flight crews [21]. Controllers can then be alerted to upcoming spacing problems and delegate a limited spacing 


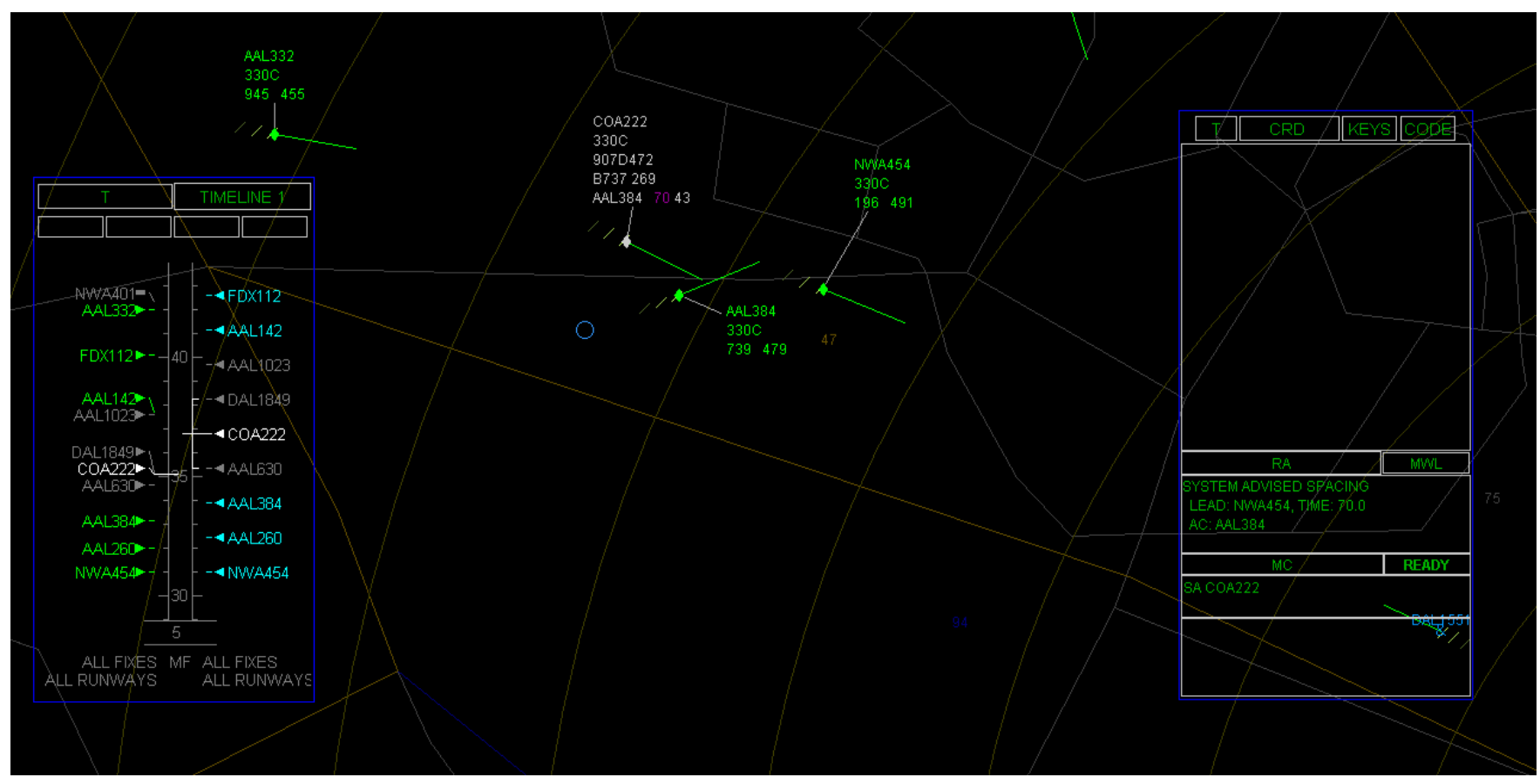

Figure 3: Protoype for DSR radar controller station. TFM generated time constraints and compliance are displayed in timeline, spacing advisories in the data tag, and local spacing conformance graphically.

task to flight crews equipped with ASAS automation.

\section{Ground side}

Figure 7 depicts a prototypical Display System Replacement (DSR) high altitude radar controller position integrating trajectory orientation and relative spacing. The timeline on the left side of the figure displays estimated times of arrival (ETA) on the left side and STAs on the right side. These times are referenced to a meter fix used to meter traffic from the downstream low altitude sector into TRACON airspace. By adjusting aircraft trajectories to meet these times the traffic gets preconditioned for merging at the meter fix. This reduces workload for the low altitude controller and allows aircraft to absorb delays in the more fuel efficient and less noise sensitive cruise phase of flight. The enhanced flow conformance feedback provided by the time line can enable trajectory orientation in the near term. As more ground-based decision support tools like the CTAS Enroute Descent Advisor (EDA) become available controllers can get additional support in the trajectory planning task.

In the center of the display excerpt shown in figure 7 a local spacing problem between merging aircraft has to be resolved. The expanded data tag of the highlighted aircraft COA222 indicates a decision support tool suggestion to pair COA222 with AAL384 and delegate a relative spacing of 70 seconds to COA222. The current spacing is estimated at 43 seconds. A circle on the display depicts where the lead aircraft AAL384 was 70 seconds ago. This graphical indication can provide controllers with feedback about the current state of the relative spacing. The DST function that provides the spacing support is straight forward and relatively easy to implement in the near term.

\section{Air side}

Current day flight management automation can be used to follow the generated trajectories precisely. However, flight deck displays and some automation will be required to support the limit delegation. When CPDLC or other data link technologies become available, more complex trajectories can be communicated between the air and the ground. The limited delegation of relative spacing can be accomplished with ASAS. Research in Europe [2] and the US [13] [22][23] has shown the feasibility and the potential benefits of this delegation. Figure 8 shows an example cockpit display for self-spacing. 


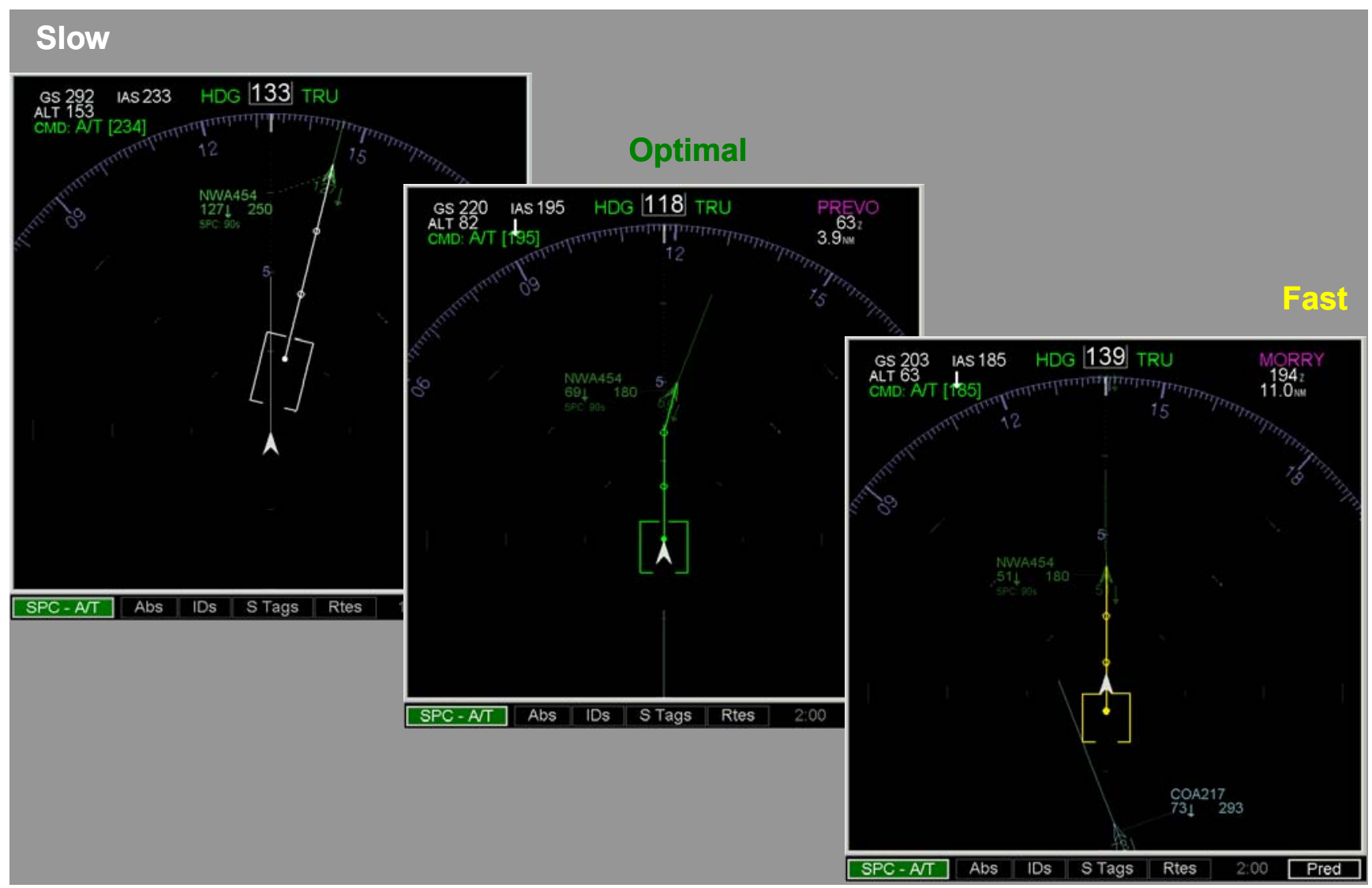

Figure 8: CDTI support for relative operations on-board the aircraft. A box indicates the desired location. The box color changes according to the current state. The command speed to fly is displayed in the upper left corner.

\section{Mid-term: Incremental integration of new CNS and DSTs and controlled paradigm shift}

The concept provides for the incremental integration of CNS technologies as well as groundside and flight deck automation as their underlying algorithms mature and human-automationintegration issues are thoroughly researched and addressed. These technologies enable advanced operational concepts like DAG-TM CE6 "trajectory negotiation" and CE 5 "free maneuvering". Concepts like CE 5 however require a paradigm shift from current day operations, because separation responsibility is delegated from the controller to the flight deck.

One of the fundamental advantages of the proposed concept that combines trajectory orientation and limited delegation of spacing operations early in the near-term is that any paradigm shift can occur slowly. It can be controlled by operational procedures rather than dictated by new automation. The initial delegation of speed control to accomplish the spacing task along a known trajectory will give flight crews and controllers initial experience with new execution/monitoring roles. The delegation of more degrees of freedom like local heading or altitude control relative to other aircraft within tolerances provides controller and flight crew feedback and operational performance data in a safe environment at different levels of automation integration and separation authority. At the same time trajectory planning can migrate to a distributed process of air and ground operations facilitated by advances in CDTI technologies [23]. 


\section{Far-term: Autonomous trajectory planning and spacing of free maneuvering aircraft}

In the far term the trajectory planning and flight execution layers could be handled autonomously by properly equipped aircraft as long as the trajectories comply with TFM constraints and are communicated to other participants. Like the ground side in the near and mid term, the flight deck trajectories only need to be de-conflicted with minimal separation buffers, because the ASAS layer is still intact to support relative spacing operations. This means that an aircraft has to detect the need for resolving a local spacing problem autonomously and switch to the ASAS mode while the problem exists. Controllers support lesser equipped aircraft with trajectory planning tasks and delegate limited spacing tasks or issue radar vectors as can be supported by the available aircraft equipage.

\section{Airspace}

The use of the concept is not restricted to any particular type of airspace. It is likely most powerful, if used during all phases of flight. Trajectory planning starts pre-flight and can be updated throughout the flight. Aircraft can be spaced behind each other to expedite departures, continue to follow their trajectories in en route airspace while occasionally slowing down or speeding up temporarily to avoid other aircraft, and use self-merging and spacing when entering congested arrival airspace. Self-spacing can be maintained until the lead aircraft has landed. Whenever necessary or desirable the trajectories can be modified to accommodate new traffic flow requirements, weather conditions, or airline scheduling constraints.

While concept use throughout the entire airspace might be desirable, significant benefits may be gained by implementing it initially in only very congested airspace and the surrounding sectors. High and low altitude arrival sectors that have to handle high traffic loads are particularly compelling early application candidates. High altitude sector controllers can set up trajectories preparing aircraft for the low altitude merge into the terminal approach airspace, and pair up aircraft that will follow each other into the TRACON. The low altitude controller can then issue self-merging and additional spacing clearances to fine-tune the feed into approach sectors taking approach controllerrequested spacing preferences into account.

\section{Research}

Research is planned at NASA Ames Research Center to further investigate this concept. Initial concept evaluations will be conducted in fast time and real time with the Multi Aircraft Control System (MACS) and advanced CDTI single piloted simulators. The DAG-TM simulation environment described in [25] and [26] will be used for evaluating the concept with pilot and controller participants. Recent early tests at Ames Research Center with researchers acting as pilots and controllers were promising in terms of efficiency, safety and workload.

\section{Concluding Remarks}

Four-D trajectory and Airborne Separation Assistance System operations are often deemed incompatible concepts because the former is by its nature strategic, the later tactical. The air traffic concept proposed in this paper is defined as

1. Use trajectory-based operations to create efficient, nominally conflict-free trajectories that conform to traffic management constraints and,

2. maintain local spacing between aircraft with airborne separation assistance.

This concept integrates the two approaches, showing a potential for maintaining high safety and improving efficiency over today's sector-based systems. The concept can be implemented evolutionarily, and a paradigm shift by air traffic controllers and pilots can occur slowly. It can build on existing tools and strategies, can provide immediate and emergent benefits, and is compatible with advanced DAG-TM concepts. A key advantage of the concept is that the full benefit of trajectory-based operations can be realized without having to generate completely de-conflicted routes with 'buffers' for prediction uncertainty. A second advantage, given that flight crews monitor 'local' situations in addition to ground controllers, is a further level of operational safety - a second set of eyes. 


\section{References}

[1] Eurocontrol, 1999, PHARE Research

Programme. http://www.eurocontrol.int/phare

[2] Eurocontrol, 2003, CoSpace

Delegation of spacing tasks from air traffic

controller to flight deck.

http://www.eurocontrol.fr/projects/freer

[3] AATT, 1999, Concept Definition for Distributed

Air/Ground Traffic Management (DAG-TM), Version 1.0, NASA AATT Project Office, Ames

Research Center, Moffett Field, CA. and http://humanfactors.arc.nasa.gov/ihh/DAG WEB

[4] Green, Steve. M., K. D. Billimoria, and M. G. Ballin 2001, Distributed Air/Ground Traffic Management for En Route Flight Operations ATC Quarterly Vol. 9(4), pp 259-285, Arlington, VA

[5] Graham, R., E. Hoffmann, C. Pusch, and K. Zeghal, 2002, 2003, Absolute versus Relative Navigation: Theoretical Considerations from an ATM Perspective, ATM2003, FAA/Eurocontrol R\&D Seminar, Budapest, Hungary

[6] Prevot T., P. Lee, T. Callantine, N. Smith, and E. Palmer, 2003, Trajectory-Oriented Time-Based Arrival Operations: Results and Recommendations, ATM2003, FAA/Eurocontrol R\&D Seminar, Budapest, Hungary

[7] Prevôt, T., V. Battiste, E. Palmer, and S. Shelden, 2003, Air Traffic Concept Utilizing 4D Trajectories and Airborne Separation Assistance, AIAA-2003-5770, Reston, VA.

[8] J. Villiers, Perspectives for Air Traffic Control for Advanced Phases of Automation - the Method of Layers (in French: Perspectives pour le contröle de la circulation aérienne dans les phases avancées d'automatisation - la méthode des filtres), Navigation $n^{\circ}$ 61, Paris, January 1968

[9] Haraldsdottir A., E. G. Schoemig, R. W. Schwab, M. K. Singleton, A. H. Sipe, P. A. van Tulder (2003) Boeing Capacity-Increasing ATM Concept for 2020 ATM2003, FAA/Eurocontrol R\&D Seminar, Budapest, Hungary

[10] Erzberger, H., T.J. Davis, and S.M. Green, 1993, Design of Center-TRACON Automation System, AGARD Meeting on Machine Intelligence in Air Traffic Management, Berlin, Germany, May, and http://ctas.arc.nasa.gov
[11] Davis, T.J., H. Erzberger, and H. Bergeron, 1989, Design of a Final Approach Spacing Tool for TRACON Air Traffic Control, NASA Technical Memorandum 102229, Ames Research Center, 1989.

[12] Green S. M. and R Vivona, 2001, En Route Descent Advisor Concept for Arrival Metering AIAA2001-4144, AIAA GNC Conference,

[13] Lee P., J. Mercer, T. Prevot, N. Smith, V. Battiste W. Johnson R. Mogford, and E. Palmer, 2003, Free Maneuvering, Trajectory Negotiation, and Self-Spacing Concepts in Distributed AirGround Traffic Management, ATM2003, FAA/Eurocontrol R\&D Seminar, Budapest, Hungary.

[14] Erzberger, H. and R.A. Paielli, 2002, Concept for next generation air traffic control system. Air Traffic Control Quarterly, Vol. 10(4) 355-378. Arlington, VA.

[15] Leiden, K.J and S. M. Green 2000, Trajectory Orientation: A Technology-Enabled Concept Requiring a Shift in Controller Roles and Responsibilities. 3rd USA/Europe ATM R\&D seminar, Napoli, Italy, June

[16] Petre, E. 1991 Time based Air Traffic Control in an Extended Terminal Area: A survey of Such Systems, Europcontrol Division E1, Doc. 912009, European Organisation for the Safety of Air Navigation, Eurocontrol, Rue de la Loi 72

[17] Swenson, H. N., T. Hoang, S. Engelland, D. Vincent, T. Sanders, B. Sanford, and K. Heere, Design and Operational Evaluation of the Traffic Management Advisor at the Fort Worth Air Route Traffic Control Center, 1st USA/Europe Air Traffic Management R\&D Seminar, Saclay, France, June, 1997

[18] Farley, Todd, Foster, John, Hoang, Ty, Lee, K. 2001, A Time-Based Approach to Metering Arrival Traffic to Philadelphia, AIAA 2001-5241.

[19] Green, S., 2000, En Route Spacing Tool: Efficient Conflict-free Spacing to Flow-Restricted Airspace, ATM2000 3rd USA/Europe Air Traffic Management R\&D Seminar, Napolo, JUne 2000.

[20] Callantine, T., Prevot, T., Smith, N., and Palmer, E. (2001). Simulation of CTAS/FMS Air Traffic Management. Proceedings of the 4th 
USA/Europe Air Traffic Management Research and Development Seminar, Air-Ground Cooperation Track, Santa Fe, NM,

[21] Crane, B, T. Prevot and E. Palmer. (1999)

Flight Crew Factors for CTAS/FMS Integration in the Terminal Airspace. NASA Technical

Memorandum 2000-209607, NASA Ames Research Center, Moffett Field, CA.

[22] Lohr G., R. M. Oseguera-Lohr, T. S. Abbott, W. R. Capron (2003) Flight Evaluation of a Timebased Airborne Inter-Arrival Spacing Tool ATM2003, FAA/Eurocontrol R\&D Seminar, Budapest, Hungary

[23] Shelden, S. 2001, Evaluation of a Terminal Area In-Trail Approach Spacing, Project and Study. NASA Technical Paper CR-2001-210920, NASA Ames Research Center, Moffett Field, CA.

[24] Johnson, W., V. Battiste, and S. Bochow, 1999, A cockpit display designed to enable limited flight deck separation responsibility, SAE Technical Paper 1999-01-5567, SAE International, Warrendale, PA.

[25] Prevôt, T., E. Palmer, N. Smith, and T. Callantine, 2002, A multi-fidelity simulation environment for human-in-the-loop studies of distributed air ground traffic management, AIAA2002-4679, Reston, VA.

[26] Prevôt, T., S. Shelden, E. Palmer, W.Johnson, V. Battiste, N. Smith, T. Callantine, P. Lee and J. Mercer 2003, Distributed air/ground traffic management simulation: results, progress and plans, AIAA-2003-5602, Reston, VA.

\section{Acknowledgements}

The research providing results and insight for development of this concept owes its success to many dedicated individuals including at NASA's Ames Research Center - Nancy Smith, Todd Callantine, Paul Lee, Joey Mercer, Walter Johnson, the Flight Deck Research Group, Sandy Lozito and her research team, Dave Encisco and the Airspace Operations Lab support staff, the MACS development team, and the Advanced Concepts Flight Simulator support staff. We greatly appreciate the support of the Air Line Pilots Association, the National Air Traffic Controllers Association, the FAA's Air Traffic Services office.
Funding for this work was provided by the Advanced Air Transportation Technologies (AATT) project office of NASA's Airspace Systems Program. 